\title{
Correction to: Assessing an efficient hybrid of Monte Carlo technique (GSA-GLUE) in Uncertainty and Sensitivity Analysis of vanGenuchten Soil Moisture Characteristics Curve
}

\author{
Samaneh Etminan ${ }^{1} \cdot$ Vahidreza Jalali $^{1,2}$ (D) Majid Mahmoodabadi $^{3} \cdot$ Abbas Khashei siuki $^{4} \cdot$ Mohsen Pourreza Bilondi $^{4}$ \\ Published online: 5 February 2021 \\ (C) Springer Nature Switzerland AG 2021
}

\section{Correction to: Computational Geosciences https://doi.org/10.1007/s10596-020-10019-w}

The original version of the article unfortunately contained an error.

The word "Birjand" was inadvertently added in the second author's affiliation. The correct second author's affiliation is as below:

Department of Rangeland and watershed management, Higher Education Complex of Shirvan, North Khorasan, Iran

The original article has been corrected.

Publisher's note Springer Nature remains neutral with regard to jurisdictional claims in published maps and institutional affiliations.

The online version of the original article can be found at https://doi.org/ 10.1007/s10596-020-10019-w

Vahidreza Jalali

v.jalali@uk.ac.ir

1 Department of Soil Science, Faculty of Agriculture, Shahid Bahonar University of Kerman, Kerman, Iran

2 Department of Rangeland and watershed management, Higher Education Complex of Shirvan, North Khorasan, Iran

3 Department of Soil Science, Faculty of Agriculture, Shahid Bahonar University of Kerman, Kerman, Iran

4 Associate Professor, Department of Water Engineering, Faculty of Agriculture, University of Birjand, Southern Khorasan, Iran 